\title{
Prognostic Significance of Preoperative Serum Carcinoembryonic Antigen Varies with Lymph Node Metastasis Status in Colorectal Cancer
}

\author{
Jing Jia $\mathbb{D}^{1},{ }^{1}$ MinZhe Li $\mathbb{D},{ }^{2}$ Wenhao Teng $\mathbb{D},{ }^{3}$ Lin Wang $\mathbb{D},{ }^{1}$ Weidong Zang $\mathbb{D},{ }^{3}$ Jun Xiao $\mathbb{D},{ }^{2}$ \\ and Ying Chen $\mathbb{D}^{1}$ \\ ${ }^{1}$ Central Laboratory, Fujian Medical University Cancer Hospitale Fujian Cancer Hospital, Fuzhou, China \\ ${ }^{2}$ General Surgery Department, Beijing Chao-Yang Hospital, Capital Medical University, Beijing, China \\ ${ }^{3}$ Department of Gastrointestinal Surgery, Fujian Medical University Cancer Hospitale Fujian Cancer Hospital, Fuzhou, China
}

Correspondence should be addressed to Jun Xiao; yydst_1744@126.com and Ying Chen; fjbccy@fjmu.edu.cn

Received 20 April 2021; Revised 8 December 2021; Accepted 11 December 2021; Published 27 December 2021

Academic Editor: Dan Zhao

Copyright ( 2021 Jing Jia et al. This is an open access article distributed under the Creative Commons Attribution License, which permits unrestricted use, distribution, and reproduction in any medium, provided the original work is properly cited.

\begin{abstract}
Background. Preoperative serum level of carcinoembryonic antigen (pCEA) is generally recognized as a prognostic factor for colorectal cancer (CRC), but the stage-specific role of pCEA in colorectal cancer remains unclear. Objective. We investigated the prognostic significance of pCEA levels in different tumor stages of nonmetastatic CRC patients. Methods. Six hundred and fifteen CRC patients at stage I-III were retrospectively analyzed. All of them received curative tumor resection. The $X$-tile program was used to generate stage-specific cutoff values of pCEA for all patients and two subpopulations (lymph node-positive or -negative). The prognostic significance of pCEA was assessed using Kaplan-Meier analysis and Cox proportional hazards regression analysis. A nomogram model that combined pCEA score and clinical feature indexes was established and evaluated. Results. Two cutoff values were identified in the study population. At a cutoff value of $4.9 \mathrm{ng} / \mathrm{mL}$, a significantly higher 5 -year overall survival (OS) rate $(82.16 \%)$ was observed in the pCEA-low group $(<4.9 \mathrm{ng} / \mathrm{mL})$ compared with $65.52 \%$ in the pCEA-high group $(\geq 4.9 \mathrm{ng} / \mathrm{mL})$. Furthermore, at the second cutoff value of $27.2 \mathrm{ng} / \mathrm{mL}$, 5-year OS was found to be only $40.9 \%$. Stratification analysis revealed that preoperative serum level of pCEA was an independent prognostic factor $(\mathrm{OR}=1.991, P<0.01)$ in the subpopulation of lymph node metastasis (stage III) patients, and the relative survival rates in the pCEA-low $(\leq 4.9 \mathrm{ng} / \mathrm{mL}), \mathrm{pCEA}$-medium $(4.9-27.2 \mathrm{ng} /$ $\mathrm{mL})$, and pCEA-high $(\geq 27.2 \mathrm{ng} / \mathrm{mL})$ groups were $73.4 \%, 60.5 \%$, and $24.8 \%$, respectively $(P<0.05)$. However, no such effect was observed in the lymph node nonmetastasis (stage I and II) subgroup. The established nomogram showed acceptable predictive power of the 5-year OS rate (C-index: 0.612) in lymph node-positive CRC patients, with an area under the curve value of 0.772 , as assessed by ROC curve analysis. Conclusions. Pretreatment serum CEA levels had different prognostic significance based on the lymph node metastasis status. Among stage III CRC patients, pCEA was an independent prognostic factor. Five-year OS rates could be predicted according to the individual pCEA level at the different cutoff values.
\end{abstract}

\section{Introduction}

Carcinoembryonic antigen (CEA) is a tumor marker in gastrointestinal cancers, particularly colorectal cancer (CRC). It belongs to a superfamily of glycoproteins expressed on cell membranes that play an important role in cell recognition and adhesion. Since it was first described in $1965[1,2]$, the biological function and clinical significance of CEA in CRC have been intensively investigated [3-5].
It is generally accepted that preoperative CEA (pCEA) is not suitable for screening or diagnostic purposes in CRC [6]. However, the prognostic role of pCEA in CRC was suggested by many studies. Based on these findings, two important issues were published in 2000. First, the American Joint Committee on Cancer proposed that pCEA can be added to the TNM staging system as an additional stratification factor for CRC on the basis of the presence or absence of a preoperative serum level of pCEA $\geq 5 \mathrm{ng} / \mathrm{mL}$ [7]. Second, the 
College of American Pathologists Expert Groups included pCEA concentration as a category I prognostic marker for CRC [8]. Six years later, two international independent organizations, the American Society of Clinical Oncology and the European Group on Tumor Markers, both recommended pCEA as a prognostic indicator in CRC $[9,10]$.

Despite a consensus being reached on the role of pCEA in $\mathrm{CRC}$, the practical application of pCEA as a routine tool was still obstructed by many uncertainties. One was the definition of an "elevated" pCEA level. Because the elevation of preoperative pCEA was associated with the development of tumor stage, the traditional use of a single pCEA cutoff value for prognostic assessment may not be suitable for all CRC patients at other stages [11-13]. Many recent studies suggested the significance of using different cutoff values for prognostic evaluation $[12,14,15]$. On the basis of this hypothesis, we performed an evaluation of different cutoff values of pCEA in subgroups of CRC patients with or without lymph node metastasis, hoping to further develop the application value of this traditional tumor marker in clinical practice.

\section{Materials and Methods}

2.1. Patients. Six hundred and fifteen eligible patients who were diagnosed with nonmetastatic resectable CRC during the period January 2010 to December 2013 at Fujian Cancer Hospital were recruited. All patients underwent surgery. Data for preoperative serum CEA level, clinicopathological features, and individual characteristics from each patient were retrospectively retrieved from the patient records within the hospital database.

In keeping with the $8^{\text {th }}$ edition of the TNM classification system [16], the inclusion criteria included the following: (1) $\mathrm{pT}_{\mathrm{X}} \mathrm{N}_{\mathrm{X}} \mathrm{M} 0$ resectable CRC; (2) adenocarcinoma confirmed by histopathological examination; (3) physical fitness suitable for surgery; and (4) without receiving any type of adjunctive therapy. The exclusion criteria included the following: (1) over 90 years old; (2) with preexisting or other concomitant cancers; (3) distant metastatic diseases; (4) noncurative resection; (5) multiple primary malignancies; (6) died within 30 days after surgery.

All patients were followed up by letter or telephone interview. The last follow-up was conducted in January 2018, and the median follow-up period was 57 months (range, 7-90 months). (1) Over 85 years of age $(n=21)$; (2) with previous or other concomitant cancers $(n=11)$; (3) distant metastatic disease $(n=31)$; (4) noncurative resection $(n=12)$; (5) multiple primary malignancies $(n=11)$; $(6)$ mortality within $30 \mathrm{~d}$ after surgery $(n=0)$. The process diagram of this article is as Line 1.

2.2. Statistical Analysis. Statistical analyses were conducted using SPSS software version 19.0. The distributions of baseline characteristics were compared using either unpaired $t$-test or ANOVA test. The cutoff values of pCEA were determined and analyzed using the $R$ (survival ROC) and $X$ tile program, which identified the cutoff with the minimum
$P$ value from log-rank $\chi^{2}$ statistics for the categorical pCEA in terms of survival. Meaningful factors were calculated using the logistic regression method extracted for further analyses. The overall cumulative probability of survival was calculated using the Kaplan-Meier method, and differences were evaluated using the log-rank test.

To evaluate the numerical weight of each factor, particularly the influence of pCEA, for predicting the long-term clinical outcome, a nomogram model integrating pCEA level and the AJCC 8th staging system were developed using $R 4.0$ software. The concordance index (C-index), receiver operating characteristic (ROC) curve, and internal calibration plot were further used to evaluate predictive performance. $P$ values of $<0.05$ were considered statistically significant.

\section{Results}

3.1. Clinicopathological Characteristics of Study Subjects. Among 615 CRC patients, there were 87 (14.15\%) at stage I, $237(38.54 \%)$ at stage II, and $291(47.32 \%)$ at stage III. The 1-, 3 -, and 5-year overall survival (OS) rates were 99.35\%, $97.56 \%$, and $74.30 \%$, respectively. The distribution of pCEA level ranged from 0.3 to $266.2 \mathrm{ng} / \mathrm{mL}$. Detailed information of recruited patients is summarized in Table 1.

\subsection{Prognostic Significance of pCEA in Nonmetastatic CRC} Patients. The prognostic significance of CEA was analyzed according to the $X$-tile program. Two cutoff values were detected. At the first cutoff value of $4.9 \mathrm{ng} / \mathrm{mL}$, a significantly higher 5-year OS rate (82.16\%) was observed in the CEA-low group $(<4.9 \mathrm{ng} / \mathrm{mL})$ as compared with $65.52 \%$ in the CEAhigh group $(\geq 4.9 \mathrm{ng} / \mathrm{mL})(P<0.05)$ (Figure 1$)$.

At the second cutoff value of $27.2 \mathrm{ng} / \mathrm{mL}$, a significantly worse prognosis population was identified in patients with CEA levels more than $27.2 \mathrm{ng} / \mathrm{mL}$, with a 5 -year OS rate of only $40.9 \%$. Thus, our data could stratify nonmetastatic CRC patients with different survival risks, i.e., CEA-low $(<4.9 \mathrm{ng} /$ $\mathrm{mL})$, CEA-medium $(4.9-27.2 \mathrm{ng} / \mathrm{mL})$, and CEA-high $(>27.2 \mathrm{ng} / \mathrm{mL}$ ) groups. The relative 5-year survival rates of these three subgroups were $82.6 \%, 67.5 \%$, and $40.9 \%$, respectively $(P<0.05)$ (Figure 2$)$.

Multivariate analyses revealed $T_{4}$ stage, $N_{2}$ stage, and CEA level were independent prognostic factors for nonmetastatic CRC (Table 2).

\subsection{Prognostic Significance of pCEA in Subpopulations with} Different Lymph Node Metastasis Statuses. To address the question of whether there was a difference between subgroups of patients with or without lymph node metastasis regarding the prognostic performance of pCEA, survival analyses were further conducted using the created cutoff values. In the subgroup of lymph node metastasis ( $\mathrm{pN1} 1-2)$, the 5-year survival rates of the three subgroups, pCEA-low $(\leq 4.9 \mathrm{ng} / \mathrm{mL}), \mathrm{pCEA}-\mathrm{medium}(4.9-27.2 \mathrm{ng} / \mathrm{mL})$, and pCEAhigh $(\geq 27.2 \mathrm{ng} / \mathrm{mL})$, were significantly different at $73.4 \%$, $60.5 \%$, and $24.8 \%$, respectively $(P<0.01)$ (Figure $3(\mathrm{a})$ ). However, no significant differences were observed in the 
TABLE 1: Demographic data of the 615 patients with CRC, $n(\%)$

\begin{tabular}{lccc}
\hline Characteristics & $(\mathrm{n}=615)$ & $\%$ & pCEA level $(\mathrm{ng} / \mathrm{mL}), \mathrm{median}(\mathrm{min}$ to $\mathrm{max})$ \\
\hline Age (years) & $58.00(20-87)$ & & $3.85(0-266.2)$ \\
\hline Gender & 229 & 37.24 & $3.48(0.3-266.2)$ \\
Female & 386 & 62.76 & $4.01(0.5-224.7)$ \\
Male & & & $3.96(0.6-77.25)$ \\
Location & 117 & 19.02 & $3.34(0.43-181.1)$ \\
Right & 151 & 24.55 & $4.2(0.3-266.2)$ \\
Left & 347 & 56.42 & $2.49(0.5-32.2)$ \\
Rectum & & & $3.36(0.3-224.7)$ \\
TNM stage & 87 & 14.15 & $5.0(0.43-266.2)$ \\
I Stage & 237 & 38.54 & $3.0(0.3-224.7)$ \\
II stage & 291 & 47.32 & $5.0(0.43-266.2)$ \\
III stage & & & \\
\hline$N$ category & 324 & 52.68 & \\
$N_{0}$ category & 291 & 47.32 & \\
$N_{1-2}$ category & &
\end{tabular}

CEA: carcinoembryonic antigen.

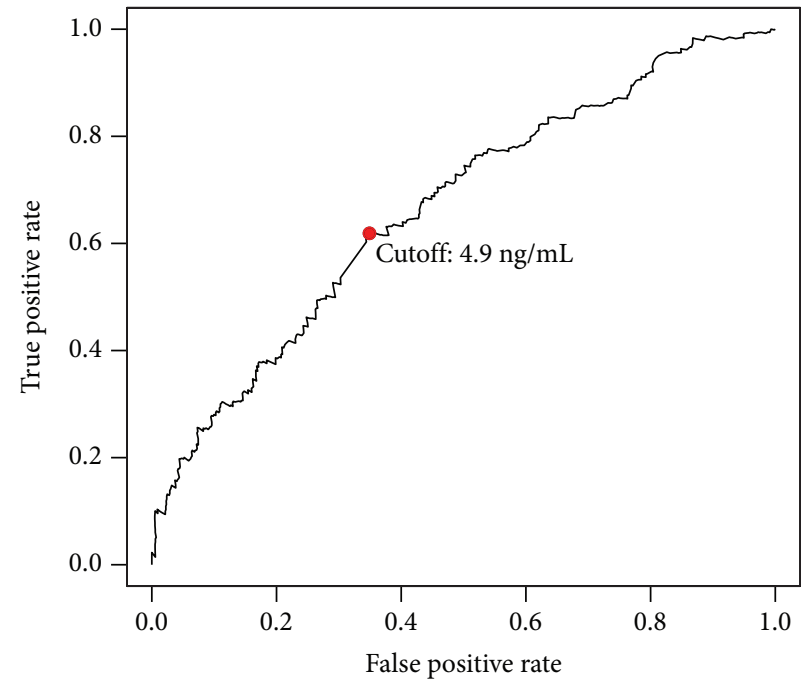

(a)

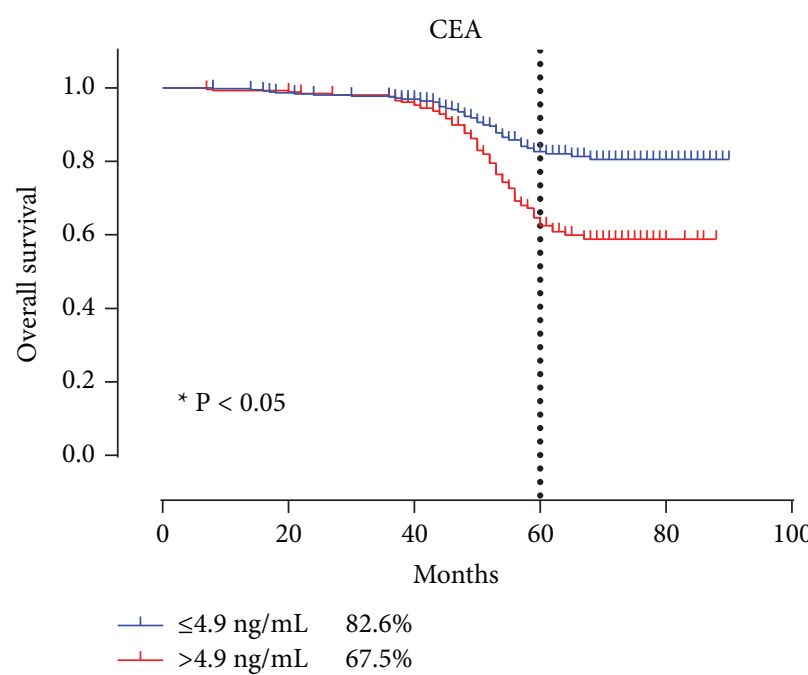

(b)

FIgURE 1: Survival analysis of CRC patients. (a) The best cutoff value calculated by R (survival ROC). (b) The 5-year survival rate of two subgroups. ROC: receiver operating characteristic curve.

lymph node nonmetastatic ( $\mathrm{pN} 0$ ) subgroup, at $89.2 \%$, $76.7 \%$, and $70.7 \%$, respectively $(P>0.05)$ (Figure $3(\mathrm{~b})$ ).

Univariant analysis further confirmed that gender $(\mathrm{OR}=1.789)$, pCEA $(\mathrm{OR}=1.875)$, and $\mathrm{T} 4$ category $(\mathrm{OR}=2.555)$ were independent factors for lymph node metastasis patients. Meanwhile, pCEA $(\mathrm{OR}=1.610)$ was independent factor for patients with negative lymph nodes (Table 3). Multivariate analysis further confirmed that pCEA $(\mathrm{OR}=1.991)$ and $\mathrm{T} 4$ category $(\mathrm{OR}=2.101)$ were independent factors for lymph node metastasis patients (Table 4).

3.4. Establishment of a Predictive Nomogram. Because pCEA level was an independent prognostic factor in stage III CRC patients, we constructed a prognostic nomogram that included gender, age, pCEA level, $T$ category, and $N$ category to predict 3- and 5-year OS in this subgroup (Figure 4(a)). The total points obtained from this model by summing the points of each variable could be used to estimate the 3- and 5-year OS rates of each patient. When the pCEA level was in the range of 5.0 to $27.2 \mathrm{ng} / \mathrm{mL}$ and pCEA was higher than $27.2 \mathrm{ng} / \mathrm{mL}$, its contribution to the prognosis of the disease was similar to $\mathrm{N}_{1}$ and $\mathrm{N}_{2}$ stage, respectively.

The internally validated Harrell's C-index was 0.612, while the area under the curve value in predicting 5-year OS reached 0.772 for this model (Figure $4(\mathrm{~b})$ ). Furthermore, the calibration plot analysis showed that the 5-year survival probability predicted by the nomogram model had optimal agreement with the actual observation (Figure 4(c)). 

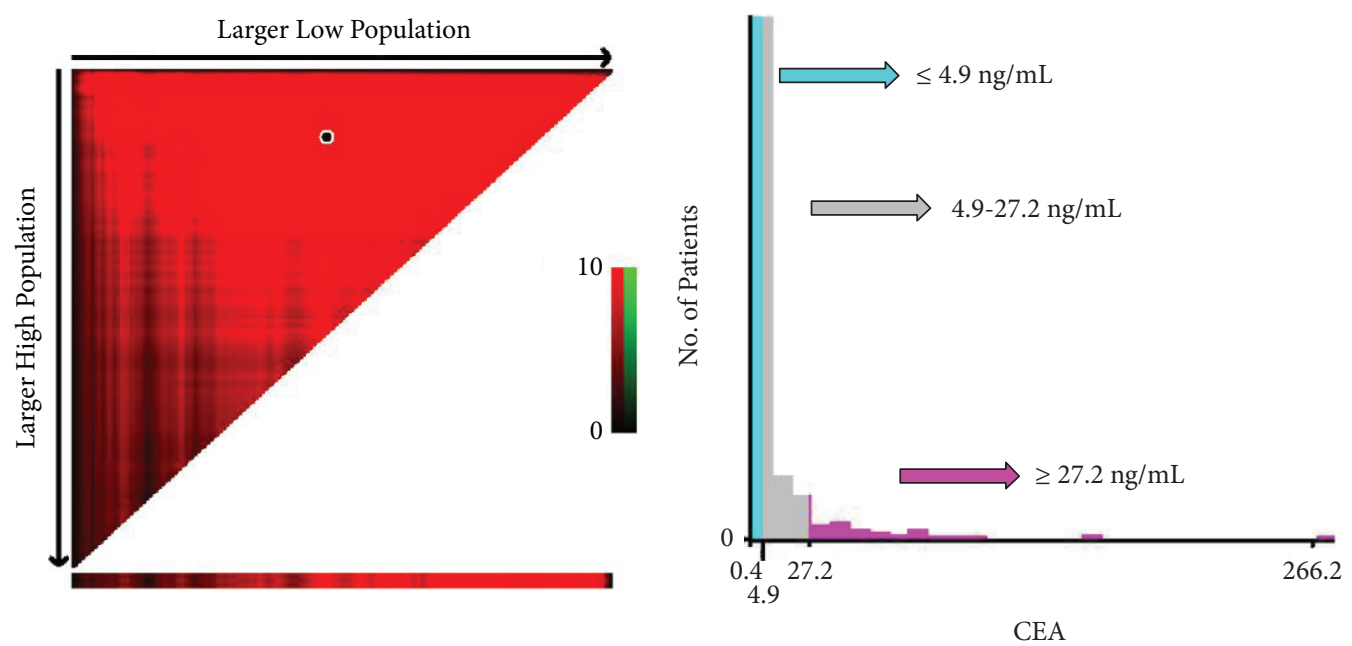

(a)

(b)

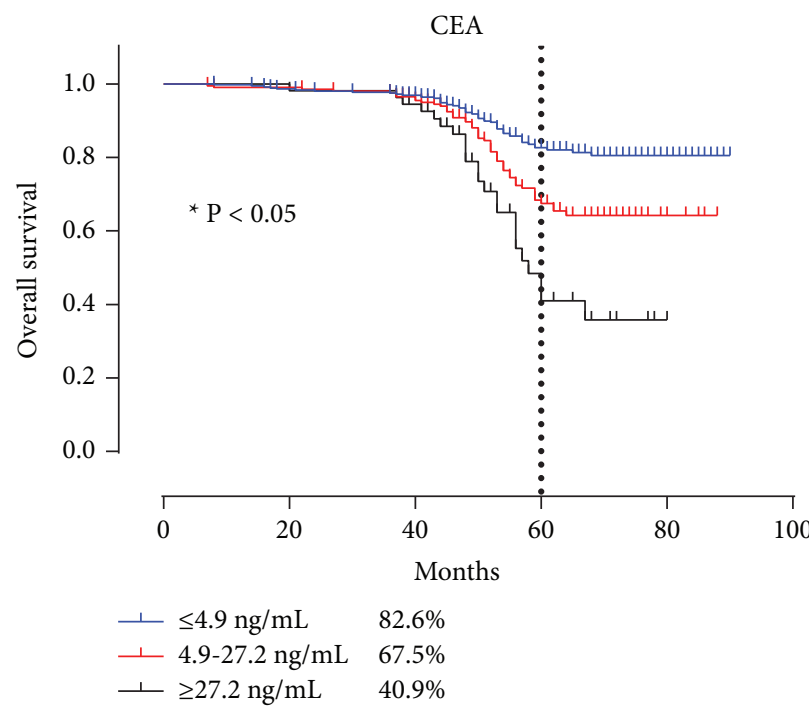

(c)

FIGURE 2: Determination of optimal cutoff values of further distinguishing high-risk groups of CRC patients. (a, b) Identification of the optimal cutoff value of pCEA by X-tile. (c) Survival analysis for low pCEA (less than 4.9), medium pCEA (4.9 to 27.2), and high pCEA (more than 27.2) groups. CEA: carcinoembryonic antigen.

TABLE 2: Multivariate analysis for CRC patients.

\begin{tabular}{|c|c|c|c|c|}
\hline & \multirow{2}{*}{$P$ value } & \multirow{2}{*}{ OR } & \multicolumn{2}{|c|}{ 95\% CI used for $\operatorname{Exp}(B)$} \\
\hline & & & Lower & Upper \\
\hline Gender & 0.447 & 0.868 & 0.603 & 1.250 \\
\hline Age & 0.234 & 1.031 & 0.747 & 1.516 \\
\hline \multicolumn{5}{|l|}{ T category } \\
\hline$T_{1}$ & 1 (reference) & & & \\
\hline$T_{2}$ & 0.107 & 1.350 & 0.937 & 1.945 \\
\hline$T_{3}$ & 0.167 & 0.365 & 0.087 & 1.523 \\
\hline$T_{4}$ & 0.000 & 1.902 & 1.686 & 3.014 \\
\hline \multicolumn{5}{|l|}{$N$ category } \\
\hline$N_{0}$ & 1 (reference) & & & \\
\hline$N_{1}$ & 0.650 & 0.630 & 0.085 & 4.643 \\
\hline $\mathrm{N}_{2}$ & 0.000 & 2.533 & 1.913 & 3.775 \\
\hline \multicolumn{5}{|l|}{ Location } \\
\hline Right & 1 (reference) & & & \\
\hline Left & 0.514 & 1.738 & 0.816 & 2.707 \\
\hline Rectum & 0.442 & 1.262 & 0.698 & 2.282 \\
\hline pCEA level & 0.000 & 1.931 & 1.396 & 2.421 \\
\hline
\end{tabular}

CEA: carcinoembryonic antigen; OR: odds ratio; $95 \%$ CI: $95 \%$ confidence interval. 


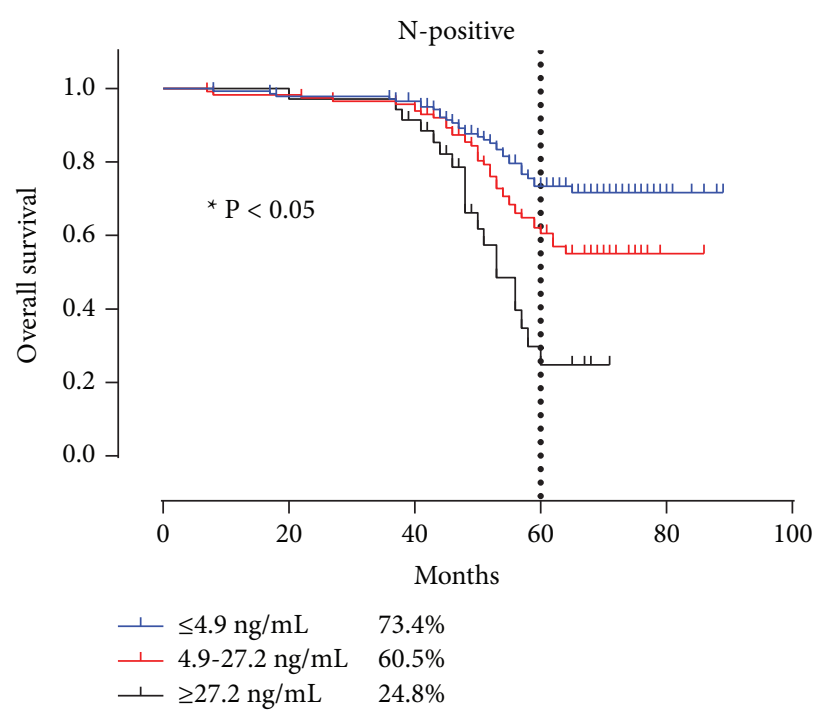

(a)

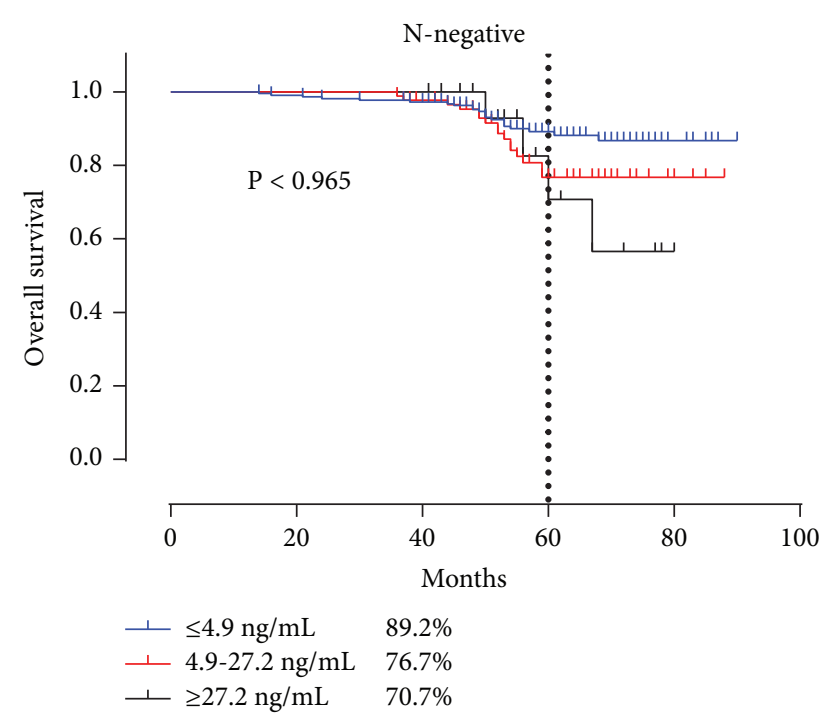

(b)

FiguRE 3: Survival curve of CRC patients according to different statuses of lymph node metastasis. (a) Lymph node metastasis group. (b) None lymph node metastasis group.

TABLE 3: Univariant analysis for CRC patients according to lymph node status.

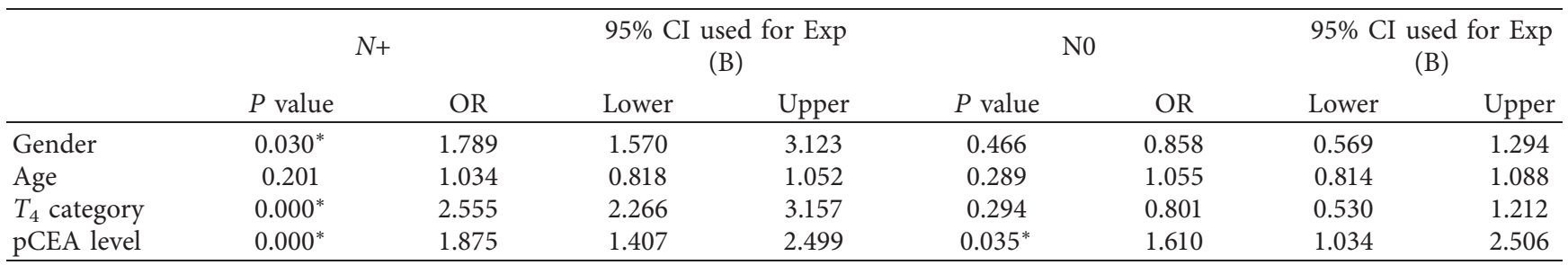

CEA: carcinoembryonic antigen; OR: odds ratio; $95 \%$ CI: $95 \%$ confidence interval; * the difference is statistically significant.

TABLE 4: Multivariant analysis for CRC patients according to lymph node status.

\begin{tabular}{|c|c|c|c|c|c|c|c|c|}
\hline & \multicolumn{2}{|c|}{$N+$} & \multicolumn{2}{|c|}{$\begin{array}{l}\text { 95\% CI used for Exp } \\
\text { (B) }\end{array}$} & \multicolumn{2}{|c|}{ No } & \multicolumn{2}{|c|}{$\begin{array}{l}\text { 95\% CI used for Exp } \\
\text { (B) }\end{array}$} \\
\hline & $P$ value & OR & Lower & Upper & $P$ value & OR & Lower & Upper \\
\hline Gender & 0.161 & 0.735 & 0.478 & 1.130 & 0.614 & 1.200 & 0.591 & 2.438 \\
\hline Age & 0.991 & 1.030 & 0.913 & 1.048 & 0.701 & 1.055 & 0.923 & 1.089 \\
\hline$T_{4}$ category & $0.000^{*}$ & 2.101 & 1.736 & 3.321 & 0.453 & 0.559 & 0.122 & 2.558 \\
\hline pCEA level & $0.000^{*}$ & 1.991 & 1.471 & 2.742 & 0.326 & 1.005 & 0.995 & 1.015 \\
\hline
\end{tabular}

CEA: carcinoembryonic antigen; OR: odds ratio; 95\% CI: 95\% confidence interval; * the difference is statistically significant.

\section{Discussion}

Considering that high preoperative CEA levels were closely correlated with tumor load, pCEA is generally identified as a biomarker for indicating multiple cancers [17]. However, the clinical prognostic value of pCEA in CRC remains unclear. There have been many discussions in previous studies on how to practically use pCEA level as a prognostic factor in CRC cases and whether the lymph node metastasis status will affect the significance of pCEA.

To address this, the current study collected 615 CRC cases based on similar endpoints. According to the CRC patients' survival results, pCEA level was found to be an independent prognostic factor for nonmetastatic CRC patients. Two cutoff values were detected in this study, accompanied by a significant prognostic outcome. The first cutoff was $4.9 \mathrm{ng} / \mathrm{mL}$, which is similar to the conventional level of $5.0 \mathrm{ng} / \mathrm{mL}$ as used previously. The second cutoff was $27.2 \mathrm{ng} / \mathrm{mL}$, which indicated a much worse 5-year OS. These findings are in line with the view of Park et al. that preoperative CEA levels could be used as a stratification parameter for identifying subsets of CRC patients with different prognostic outcomes [18].

Previous studies are unclear about the common effect of CEA and lymph node metastasis in predicting prognosis [19-21]. This problem may be related to the fact that subgroup analyses were not performed in these studies or that they used single cutoff values for pCEA. As the TNM stage of 


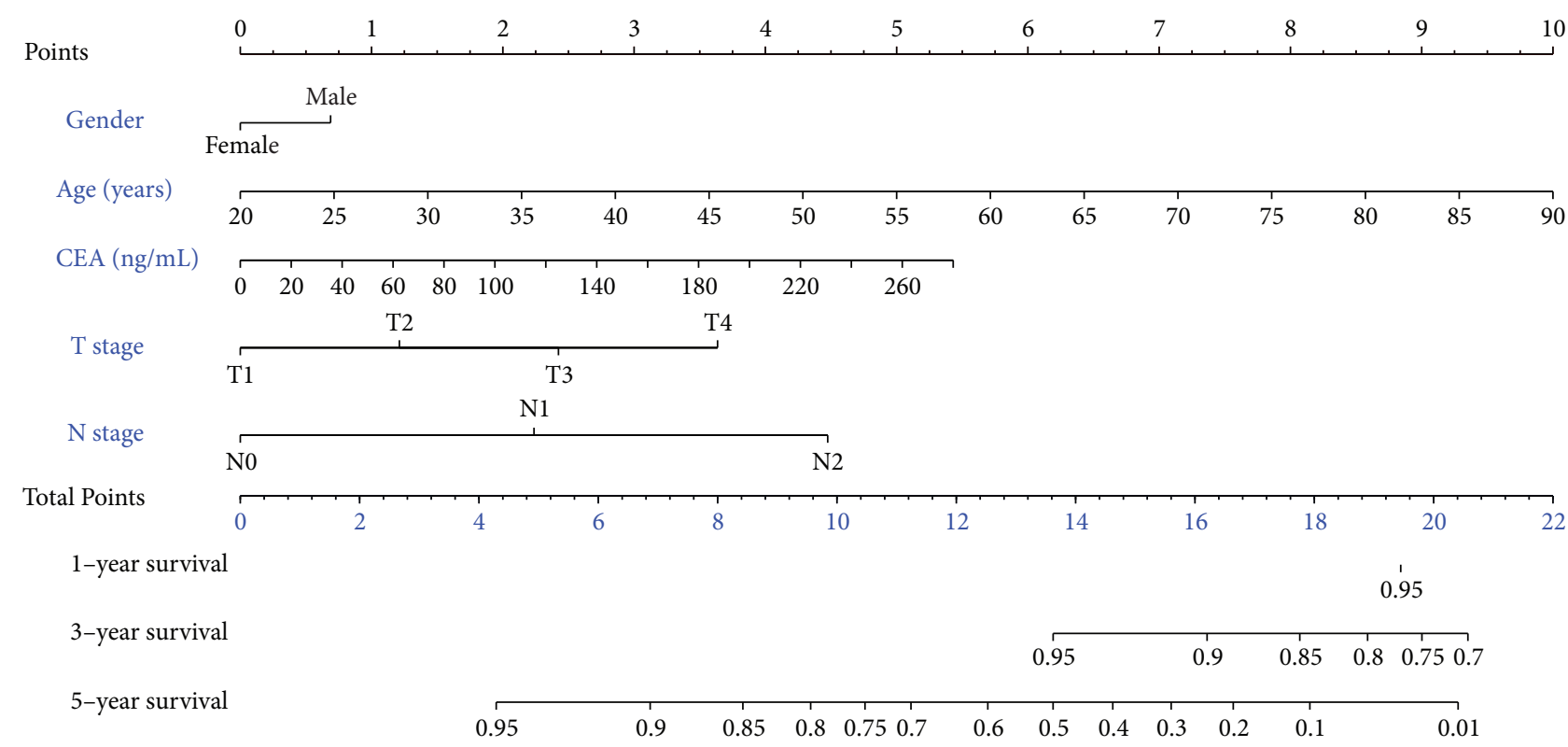

(a)

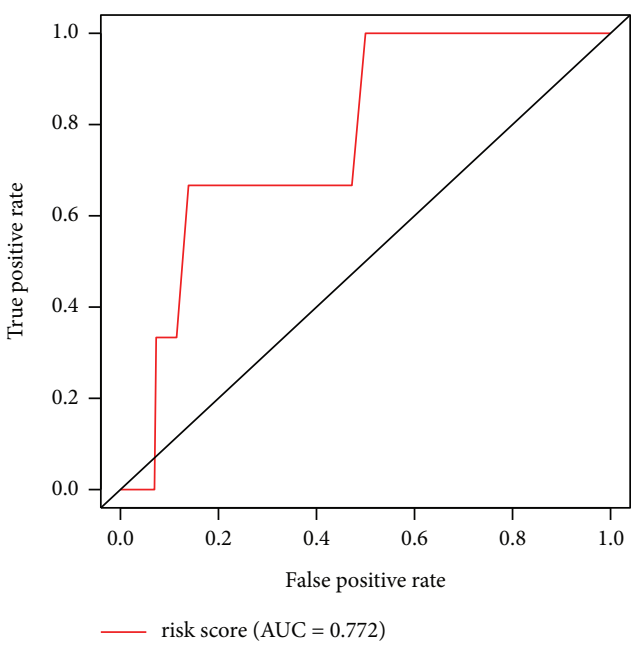

(b)

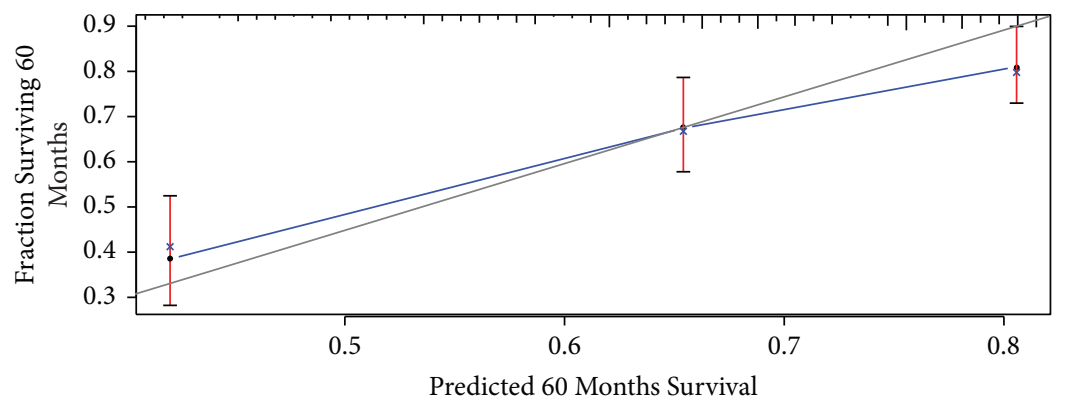

(c)

FIGURE 4: Integration model for predicting the 5-year survival probability using the nomogram. (a) The 5-year probability of death for a patient is located on the total points axis (bottom) by summing up the total points assigned to each variable at the scales shown above, as indicated with the lines drawn downward to each axis. (b) The result of ROC curves. (c) The calibration curve for the new nomogram model for 5 years. Gray: ideal model; blue: the new nomogram model. CEA: carcinoembryonic antigen; ROC: receiver operating characteristic curve.

CRC increases, its tumor burden becomes heavier, which may also enable CEA to more accurately reflect the actual condition of the patient [22]. However, at earlier stages of clinical cases, there are many confounding factors, and thus pCEA may not be able to accurately assess tumor burden [23]. Therefore, this study divided CRC cases into subgroups according to the status of lymph node metastasis. Our results revealed that in patients with positive lymph nodes, pCEA level was an independent prognostic factor $(\mathrm{OR}=1.991$, $P<0.01)$. However, in the subgroup with negative lymph nodes, CEA level was not an independent prognostic factor $(P=0.326)$. This result implied that the prognostic significance of CEA in CRC differs from TNM stage. Although our data suggested that pCEA level might be closely related to lymph node metastasis, consistent with previous research [24], the underlying mechanism remains unclear.

On this topic, some previous literatures also showed a similar research endpoint [25-27]. However, the number of samples included in the research of each literature is small, and the cutoff values obtained by statistical methods are not the same thing $[28,29]$. Therefore, the research conclusions may be biased. The direction of this study is the clinical value of CEA in predicting metastatic CRC. Clinically, there are plenty of accumulated 615 CRC cases, with clear goals, strong pertinence, and reproducibility, which has clinical practical value and translational application value. In addition, most of the literatures directly use the upper limit of the normal reference value of 5.0 as the cutoff value, instead 
of being analyzed from the critical value data. This study combined CEA evaluation parameters of the clinical prognosis to calculate the cutoff point $(4.9 \mathrm{ng} / \mathrm{mL})$, which is different from the cutoff point setting in the previous literature, and the data are more reliable.

Interestingly, as shown by the nomogram model established in this study, we found that when pCEA was in the range of 5.0 to $27.2 \mathrm{ng} / \mathrm{mL}$, its contribution to 5 -year OS was equivalent to $\mathrm{N}_{1}$ staging. Moreover, a similar association was observed between pCEA levels higher than $27.2 \mathrm{ng} / \mathrm{mL}$ and $\mathrm{N}_{2}$ staging. These results are consistent with clinical observations and further support the idea that pCEA level is closely related to the status of lymph node metastasis. Therefore, through this analysis, it may be possible to initially explain the differences in previous clinical research.

Despite the current study proving the significant role of pCEA in CRC prognosis, there are some limitations. First, the inherent bias of patient selection could not be completely avoided, which could partly affect surgical outcomes. Second, the case number of patients with high serum CEA levels was relatively limited in this study, which reduced the statistical power. Third, the data collection was localized in one hospital, which suggests that the current results might not be applicable to different regions of China.

In conclusion, we demonstrated that pCEA levels can effectively predict prognosis in CRC patients with positive lymph node metastasis. When pCEA levels are higher than $4.9 \mathrm{ng} / \mathrm{mL}$, they generally indicate worse and unfavorable tumor behavior and poor prognosis.

\section{Data Availability}

The data of this paper come from the local database of Fujian Medical University Cancer Hospital and Fujian Cancer Hospital.

\section{Disclosure}

Jing Jia and Minzhe Li are co-first authors.

\section{Conflicts of Interest}

The authors declare that they have no conflicts of interest.

\section{Acknowledgments}

The authors thank H. Nikki March, PhD, from Liwen Bianji, Edanz Editing China (http://www.liwenbianji.cn/ac), for editing the English text of a draft of this manuscript. This work was supported by Fujian Science and Technology Program (Grant nos. 2017Y0020 and 2019J01192).

\section{References}

[1] P. Gold and S. O. Freedman, "Demonstration of tumorspecific antigens in human colonic carcinomata by immunological tolerance and absorption techniques," Journal of Experimental Medicine, vol. 121, no. 3, pp. 439-462, 1965.
[2] P. Gold and S. O. Freedman, "Specific carcinoembryonic antigens of the human digestive system," Journal of Experimental Medicine, vol. 122, no. 3, pp. 467-481, 1965.

[3] M. J. Duffy, "Carcinoembryonic antigen as a marker for colorectal cancer: is it clinically useful?" Clinical Chemistry, vol. 47, no. 4, pp. 624-630, 2001.

[4] M. J. Goldstein and E. P. Mitchell, "Carcinoembryonic antigen in the staging and follow-up of patients with colorectal cancer," Cancer Investigation, vol. 23, no. 4, pp. 338-351, 2005.

[5] N. Lopez and C. Peterson, "Advances in biomarkers: going beyond the carcinoembryonic antigen," Clinics in Colon and Rectal Surgery, vol. 29, no. 03, pp. 196-204, 2016.

[6] F. Carrasco-Pena, E. Bayo-Lozano, M. Rodriguez-Barranco, D. Petrova, R. Marcos-Gragera, and M. C. Carmona-Garcia, "Adherence to clinical practice guidelines and colorectal cancer survival: a retrospective high-resolution populationbased study in Spain," International Journal of Environmental Research and Public Health, vol. 17, 2020.

[7] C. Compton, C. M. Fenoglio-Preiser, N. Pettigrew, and L. P. Fielding, "American Joint Committee on Cancer prognostic factors consensus conference," Cancer, vol. 88, no. 7 , pp. 1739-1757, 2000.

[8] C. C. Compton, L. P. Fielding, L. J. Burgart et al., "Prognostic factors in colorectal cancer," Archives of Pathology \& Laboratory Medicine, vol. 124, no. 7, pp. 979-994, 2000.

[9] M. J. Duffy, A. van Dalen, C. Haglund et al., “Tumour markers in colorectal cancer: European Group on Tumour Markers (EGTM) guidelines for clinical use," European Journal of Cancer, vol. 43, no. 9, pp. 1348-1360, 2007.

[10] A. Goel, "Utilizing biomarkers in colorectal cancer: an interview with Ajay Goel," Future Oncology, vol. 13, no. 28, pp. 2511-2514, 2017.

[11] Y.-Y. Huang, P.-I. Lee, M.-C. Liu, C.-C. Chen, K.-C. Huang, and A. T. Huang, "A general cutoff level combined with personalized dynamic change of serum carcinoembryonic antigen can suggest timely use of FDG PET for early detection of recurrent colorectal cancer," Clinical Nuclear Medicine, vol. 40, no. 10, pp. e465-e469, 2015.

[12] B.-K. Ahn, "Individualized cutoff value of the serum carcinoembryonic antigen level according to TNM stage in colorectal cancer," Annals of coloproctology, vol. 29, no. 3, pp. 91-92, 2013.

[13] B. Engarås, "Individual cutoff levels of carcinoembryonic antigen and CA 242 indicate recurrence of colorectal cancer with high sensitivity," Diseases of the Colon \& Rectum, vol. 46, no. 3, pp. 313-321, 2003.

[14] J. H. Kim, S. Lee, S. H. Lee et al., "Clinical significance of carcinoembryonic antigen in peritoneal fluid detected during operation in stage I-III colorectal cancer patients," Intestinal research, vol. 16, no. 3, pp. 467-474, 2018.

[15] Z. Cai, J. Xiao, X. He et al., "Accessing new prognostic significance of preoperative carcinoembryonic antigen in colorectal cancer receiving tumor resection: more than positive and negative," Cancer Biomarkers, vol. 19, no. 2, pp. 161-168, 2017.

[16] P. Chen, Z. L. Zuo, L. B. Feng et al., "Questioning the staging of tumor deposits of colorectal cancer in the eighth edition of the TNM classification: validation by prognosis," International Journal of Clinical and Experimental Pathology, vol. 12, pp. 4309-4318, 2019.

[17] G. Lech, R. Slotwinski, and I. W. Krasnodebski, "The role of tumor markers and biomarkers in colorectal cancer," Neoplasma, vol. 61, no. 01, pp. 1-8, 2014. 
[18] J. W. Huh, C. H. Kim, S. W. Lim, H. R. Kim, and Y. J. Kim, "Factors predicting long-term survival in colorectal cancer patients with a normal preoperative serum level of carcinoembryonic antigen," Journal of Cancer Research and Clinical Oncology, vol. 139, no. 9, pp. 1449-1455, 2013.

[19] B.-B. Su, H. Shi, and J. Wan, "Role of serum carcinoembryonic antigen in the detection of colorectal cancer before and after surgical resection," World Journal of Gastroenterology, vol. 18, no. 17, pp. 2121-2126, 2012.

[20] R. Takagawa, S. Fujii, M. Ohta et al., "Preoperative serum carcinoembryonic antigen level as a predictive factor of recurrence after curative resection of colorectal cancer," Annals of Surgical Oncology, vol. 15, no. 12, pp. 3433-3439, 2008.

[21] R. Lázaro-Gorines, J. Ruiz-de-la-Herrán, R. Navarro et al., "A novel carcinoembryonic antigen (CEA)-Targeted trimeric immunotoxin shows significantly enhanced antitumor activity in human colorectal cancer xenografts," Scientific Reports, vol. 9, no. 1, p. 11680, 2019.

[22] R. Partyka, A. Sandelewski, I. Lobejko, J. Kocot, P. Jalowiecki, and D. Kokocinska, "[Usefulness of evaluation of soluble fragment cytokeratin 18, carcinoembryonic antigen and gastrointestinal carcinoma-associated antigen in diagnostic of patients with colorectal cancer]. Polski merkuriusz lekarski," Organ Polskiego Towarzystwa Lekarskiego, vol. 29, pp. 128-130, 2010.

[23] Y. Kosaka, N. Minatani, Y. Tanaka et al., "Lymph node metastasis and high serum CEA are important prognostic factors in hormone receptor positive and HER2 negative breast cancer," Molecular and clinical oncology, vol. 9, pp. 566-574, 2018.

[24] S.-G. Wu, Z.-Y. He, H.-Y. Ren et al., "Use of CEA and CA15-3 to predict axillary lymph node metastasis in patients with breast cancer," Journal of Cancer, vol. 7, no. 1, pp. 37-41, 2016.

[25] T. Konishi, Y. Shimada, M. Hsu et al., "Association of preoperative and postoperative serum carcinoembryonic antigen and colon cancer outcome," JAMA Oncology, vol. 4, no. 3, pp. 309-315, 2018.

[26] G. Saito, S. Sadahiro, H. Kamata et al., "Monitoring of serum carcinoembryonic antigen levels after curative resection of colon cancer: cutoff values determined according to preoperative levels enhance the diagnostic accuracy for recurrence," Oncology, vol. 92, no. 5, pp. 276-282, 2017.

[27] Y. Nakamura, D. Shida, T. Tanabe et al., "Prognostic impact of preoperatively elevated and postoperatively normalized carcinoembryonic antigen levels following curative resection of stage I-III rectal cancer," Cancer Medicine, vol. 9, no. 2, pp. 653-662, 2020.

[28] A. R. Baqar, S. Wilkins, M. Staples, C. H. Angus Lee, K. Oliva, and P. McMurrick, "The role of preoperative CEA in the management of colorectal cancer: a cohort study from two cancer centres," International Journal of Surgery, vol. 64, pp. 10-15, 2019.

[29] S.-H. Beom, S. J. Shin, C. G. Kim et al., "Clinical significance of preoperative serum carcinoembryonic antigen within the normal range in colorectal cancer patients undergoing curative resection," Annals of Surgical Oncology, vol. 27, no. 8, pp. 2774-2783, 2020. 